\title{
Medicinal use of Sceletium: Characterization of Phytochemical Components of Sceletium Plant Species using HPLC with UV and Electrospray Ionization - Tandem Mass Spectroscopy.
}

\author{
Srinivas Patnala ${ }^{1}$, Isadore Kanfer ${ }^{2}$ \\ 1. Department of Chemistry, Rhodes University, Grahamstown, South Africa. ${ }^{2}$ Division of Pharmaceutics, \\ Faculty of Pharmacy, Rhodes University, Grahamstown, South Africa
}

Received, July 7, 2015; Revised, August 26, 2015; Accepted, August 26, 2015; Published, September 5 2015.

\begin{abstract}
Purpose. Sceletium plants have been used for its medicinal properties for centuries. However, there is a wide range of Sceletium plant species in which various alkaloidal components such as $\Delta^{7}$ mesembrenone, mesembrenol, mesembranol, mesembrenone, mesembrine hydrochloride, epimesembranol and, sceletium $\mathrm{A}_{4}$ differ between species. Hence, to ensure the quality of Sceletium products used as a medicine, it is imperative to identify the appropriate species using both botanical and chemical methods. The chemical approach to identify and characterize the phytochemical composition of a particular species facilitates the choice of species that will provide the purported therapeutic outcome. Hence, specific analytical methods to identify relevant constituents from complex matrices are necessary. Although HPLC-UV detection is commonly used to identify and estimate phytochemical content of medicinal plants, use of mass spectroscopy (MS) and tandem mass spectroscopy (MS/MS) can unequivocally confirm their presence/absence based on characteristic ions and fragmentation patterns. Methods. The various alkaloidal components were characterized by electrospray ionization (ESI) MS and MS/MS using an ionizing medium of $0.1 \%$ ammonium hydroxide in water mixed with acetonitrile. Compounds were purified and characterized for use as reference standards to identify the relevant alkaloidal constituents of several Sceletium plant species using HPLC with on-line UV-MS detection. Results. ESI-MS provided the $[\mathrm{M}+\mathrm{H}]^{+}$ions with respective $\mathrm{m} / \mathrm{z}$ values that related to the respective molecular weights 287, 289, 291, 287, 289, 324 and 291 for the above mentioned alkaloids, whereas, ESI MS/MS provided the characteristic fragment ions to confirm the structural identity of the individual alkaloids and subsequently used to confirm the presence and/or absence of specific alkaloids in various Sceletium plant samples. Conclusions. Whilst HPLC-UV detection has been a widely-used conventional analytical technique for both qualitative and quantitative analyses, the results highlight the necessity of ESI-MS detection to avoid erroneous identification of phytochemical components, particularly with mesembrine-type compounds which have closely related chemical structures.
\end{abstract}

This article is open to POST-PUBLICATION REVIEW. Registered readers (see "For Readers") may comment by clicking on ABSTRACT on the issue's contents page.

\section{INTRODUCTION}

The medicinal properties of plants and plant products are often based on anecdotal evidence and such claims are especially prominent when African traditional medicines are utilized for medicinal purposes. The quality, safety and efficacy of herbal products have long been questionable and highly controversial. Hence to ensure quality, phytochemical evaluation of pharmacologically active component(s) is critical and more often than not these components are specific to a particular species.
The use of Sceletium plants by the native Bushmen of Namaqualand for its pharmacological/therapeutic effects as a stimulant is well known and the traditional preparation Kougoed or Channa, made by a fermentation process, is purported to have an enhanced stimulant effect. It is also chewed frequently especially when thirsty, indicating that it could be a hunger and thirst suppressant.

Corresponding Author: Prof. Isadore Kanfer; Division of Pharmaceutics, Faculty of Pharmacy, Rhodes University, Grahamstown, South Africa; E-mail: izzy.kanfer@gmail.com 
These observations led to commercial interest to cultivate this plant (1). The Sceletium plant has historically also been used for its purported psychoactive properties by the Khoisan tribe of Southern Africa where reports of trade and commercial interests of this plant, date back as early as the $17^{\text {th }}$ century (2).

Although, the use of Sceletium has been investigated for its pharmacological effects in animal models (3), some of the alkaloidal components, such as mesembrine, mesembrenol and mesembrenone have been reported as being pharmacologically active and are promoted for use as therapeutic medicines that may be utilized for treating human ailments (4).

It is well known that a number of variables including species differences, harvesting time, growing conditions, storage and processing contribute to the variation in phytochemical components in plants. In light of the foregoing, the development of specific analytical methods are an important aspect of quality which will subsequently reflect on the safety and efficacy of products derived from plant material (5). Thus the use of modern instrumental methods of analysis such as high performance liquid chromatography (HPLC) coupled with mass spectrometry (MS), have been successfully applied to study phytochemical constituents in various matrices that provide specific information on structural identity. Electrospray ionization mass spectrometry (ESIMS) has been successfully applied to the analysis of plant products due to the provision of a soft ionization process of the molecules and its suitability to characterize multiple components that are usually present in plant products (5). The LCMS/MS method is reportedly more precise when used to generate characteristic fingerprinting in conditions where there is uncertainty with the identification of compounds characterized by HPLC-UV. It has been suggested that LC-MS is extremely beneficial in cases when retention times (RTs) vary during HPLC analysis and also in situations where reference standards are unavailable (6). In particular, in the case of analyzing components of Sceletium plant species that require identification of closely related mesembrine-type compounds of which some are epimers and have isobaric chemistries (7), highly specific methods are necessary.

Presently, Sceletium plants are mostly organically grown in greenhouses and cultivated on farms for commercial value and indicated for the treatment of mental disorders and also for a number of other uses such as anxiety and depression. Quality and composition of Sceletium products, until recently, were not tested by validated methods (8) and furthermore, anecdotal evidence suggests that a fermentation process is a key step in processing this plant material for better efficacy. Recent studies have shown transformation of the alkaloidal composition during the fermentation process (9).

Martin et al (10) in 1976 using MS, described the identification of Sceletium alkaloids. More recently, RP-UHPLC PDA and GC-MS methods have been used to analyze psychoactive alkaloids in Sceletium tortuosum (11). In a recent article by Smith (4), mention was made of a previous report (12) which speculated that the electron impact mass spectra of three unidentified alkaloids in Sceletium tortuosum appeared to place these compounds in the same structural category as mesembrine and $\Delta^{7}$ mesembrenone. However, it was emphasized that more comprehensive analysis is required for identity confirmation.

A short communication recently emphasized the need for rapid identification of mesembrine alkaloids in Sceletium tortuosum and described a non-aqueous capillary electrophoresis mass spectroscopy mentioning the complexity of analyzing Sceletium alkaloids (7).

To-date, ESI-MS profiles of these alkaloids by LCMS methods and especially by electrospray ionization have not been reported. Since analytical methods for specific Sceletium alkaloids using LCMS are conspicuously absent from the scientific literature, the main objective was to develop an appropriate LCMS method that can be applied for chromatographic fingerprinting that provides unequivocal identity of each moiety in various matrices including Sceletium plant material, extracts and related commercial products. Clearly, an appropriate qualitative method is a pre-requisite for the subsequent development of a quantitative assay for these components.

\section{METHODS}

\section{Reagents and standards}

HPLC grade methanol 215 and acetonitrile 200 were obtained from Romil Ltd. (Cambridge, Great Britain). Ammonium hydroxide $25 \%$ solution was acquired from Associated Chemical Enterprises 
(Pty) Ltd. (Southdale, South Africa). Water was purified using a Milli- $Q^{\circledR}$ system Millipore (Bedford, USA) and Millex $\mathrm{HV}^{\circledR}$ hydrophilic PVDF membrane filters $(0.45 \mu \mathrm{m})$. The Sceletium reference compounds $\Delta^{7}$ mesembrenone, mesembrenol, mesembrenone, mesembrine, were isolated from several Sceletium plant species whereas Sceletium $\mathrm{A}_{4}$ was isolated from Sceletium crassicaule (13). Mesembranol and epimesembranol were synthesized by reduction of mesembrine using hydrogen (14) and sodium borohydride respectively (15). The compounds were qualified by respective spectral evaluation ${ }^{1} \mathrm{H},{ }^{13} \mathrm{C}$ and $2 \mathrm{D}$ NMR and mass spectroscopy as well as X-ray crystallography for the crystalline compounds, mesembrine hydrochloride and mesembranol, the others being semi-solid/liquids (16).

\section{Preparation of standards and samples}

MS studies were performed using standard solutions using $0.5 \mathrm{mg} / \mathrm{ml}$ concentration in methanol for each of the reference alkaloids. The HPLC-UV standards and the samples were prepared as methanolic extracts using commercial products containing Sceletium plant material or powdered plant material from the aerial parts of the plant which was dried at $80{ }^{\circ} \mathrm{C}$ for 12 hours prior to sampling (8).

\section{Instrumentation}

Solvent extraction was carried out by sonication in an ultrasonic bath, Model 8845-30 (Cole-Parmer Instrument Company, Chicago, Illinois, USA). The LC-MS analyses were carried out using a Finnigan MAT LCQ ion trap mass spectrometer (Finnigan, San Jose, CA, USA) coupled on-line with a UV1000 variable-wavelength UV detector, a Spectra SYSTEM P2000 pump and an AS1000 auto sampler (Thermo Separation Products, Riviera Beach, FL, USA). The separation of alkaloids was achieved on a $150 \mathrm{~mm}$ x $4.6 \mathrm{~mm}$ i.d. A Phenomenex Luna ${ }^{\circledR} \mathrm{C}_{18}$ HPLC column packed with $5 \mu \mathrm{m}$ ODS-2 (Torrence, CA, USA). AG 135 analytical balance and Electronic Micro Balance MX-5 (Mettler Toledo, Switzerland) were used for samples and weighing standards respectively. An electronic pipette 71050XET (Biohit PLC, Helsinki, Finland) was used to prepare required dilutions of standard and sample solutions.

\section{HPLC Method}

Chromatographic separation of the alkaloids by HPLC was achieved using a binary gradient elution system at $1.0 \mathrm{ml} / \mathrm{min}$. The aqueous phase was mixed with ACN using an initial $80 \%$ changing to $50 \%$ over a period of 15 minutes. The injection volumes of the standards and samples were $20 \mu 1$ and the column eluates were monitored on-line with UV detection at $228 \mathrm{~nm}$ prior to MS detection. The analysis resulted in satisfactory resolution and retention times for all the reference standards and related components in Sceletium raw material with a total run time of 16 minutes (8).

\section{ESI-MS Method Development}

Optimisation of the ESI-MS conditions was carried out using reference methanolic solutions of the various alkaloids infused into the MS detector at $3 \mu \mathrm{l} / \mathrm{min}$ in conjunction with a solvent system consisting of aqueous $0.1 \%$ ammonium hydroxide solution/acetonitrile which was pumped at a flow rate of $0.3 \mathrm{ml} / \mathrm{min}$ using an HPLC pump. The $\mathrm{m} / \mathrm{z}$ values were ascertained based on the $[\mathrm{M}+\mathrm{H}]^{+}$ions and were further subjected to tandem MS to assess the characteristic fragmentation patterns of each reference compound (Figure 1a-r and Figure 2a-c). The optimised MS conditions utilised for detection are as follows: Capillary temperature was maintained at $240{ }^{\circ} \mathrm{C}$ and the sheath and auxiliary gas flow parameters were 80 and 20 arbitrary units, respectively. The spray voltage remained constant for all compounds at $4.5 \mathrm{kV}$. In addition to detection of the protonated molecular ions $[\mathrm{M}+\mathrm{H}]^{+}$, collision induced dissociation (CID) was performed in the MSMS mode and the resulting product ions monitored as fingerprints of each alkaloid.

\section{RESULTS}

Table 1 depicts the $m / z$ ions whereas the spectral profiles by full scan ESI-MS and tandem mass spectroscopy are indicated by reference to the relevant figures numbers shown in parentheses.

The data depicted in Table 1 were obtained using the aforementioned relevant qualified reference compounds, to establish an appropriate MS data base (method file) which was subsequently used for the routine analysis of seven Sceletium alkaloids present in plant material and associated products. 

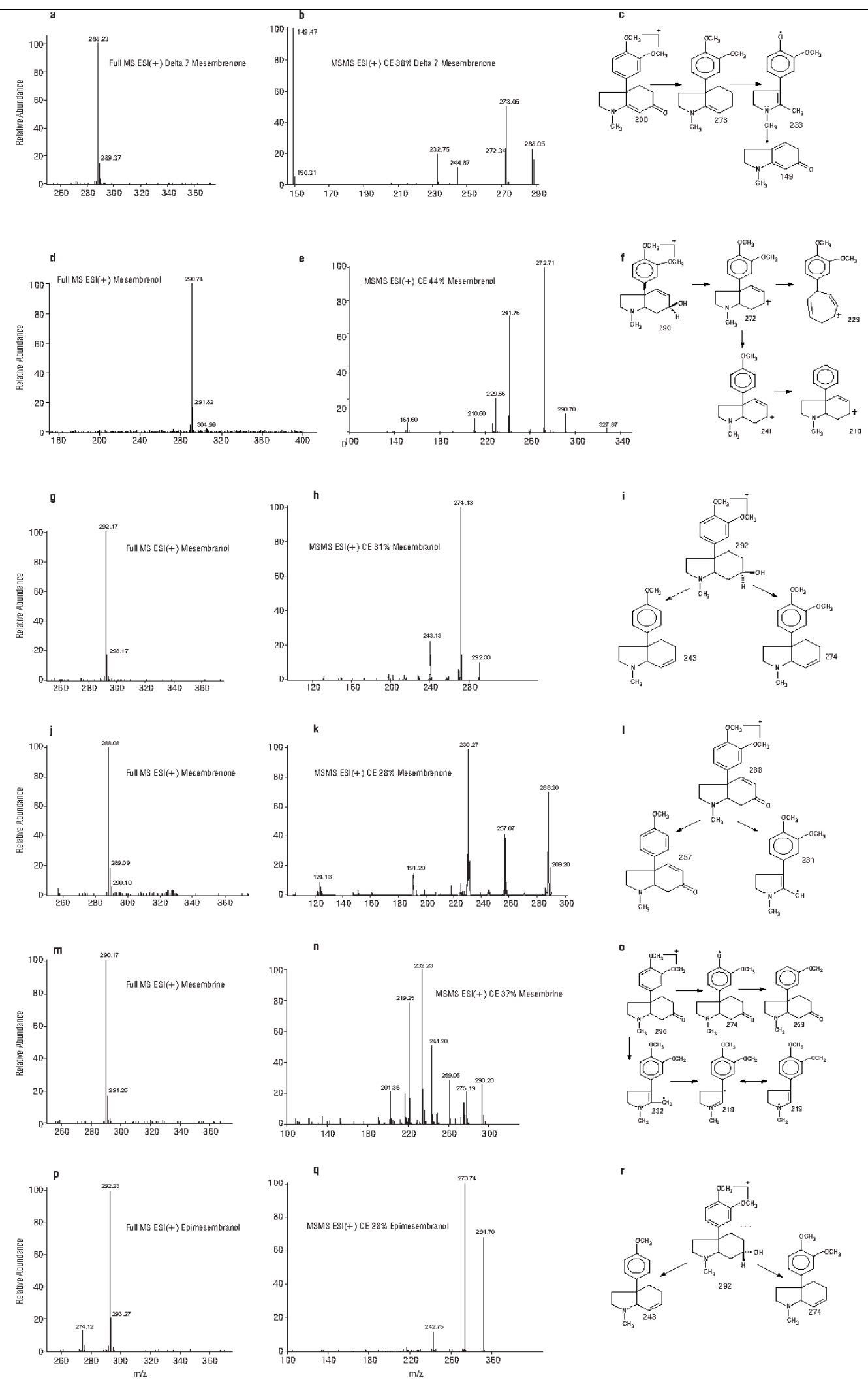

Figure 1 a-r: Mesembrine-type reference alkaloids; $\Delta^{7}$ mesembrenone (MS - 1a; MS/MS - 1b; fragmentation pattern 1c). Mesembrenol (MS - 1d; MS/MS - 1e; fragmentation pattern - 1f). Mesembranol (MS - 1g; MS/MS - 1h; fragmentation pattern - 1i). Mesembrenone (MS - 1j; MS/MS - 1k; fragmentation pattern - 11). Mesembrine (MS - 1m; MS/MS - 1n; fragmentation pattern - 1o). Epimesembranol (MS - 1p; MS/MS - 1q; fragmentation pattern - 1r). 


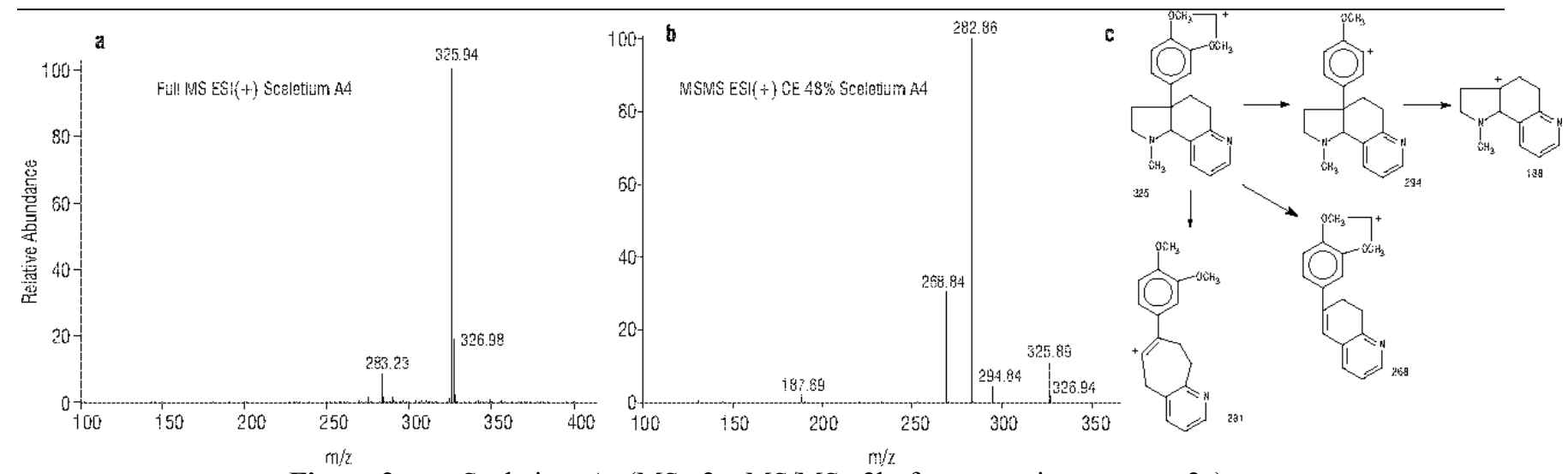

Figure 2 a-c: Sceletium $\mathrm{A}_{4}(\mathrm{MS}-2 \mathrm{a} ; \mathrm{MS} / \mathrm{MS}-2 \mathrm{~b}$; fragmentation pattern -2c).

\begin{tabular}{|c|c|c|c|c|}
\hline Alkaloid & $\begin{array}{l}\text { Molecular } \\
\text { mass }\end{array}$ & $\begin{array}{l}{[\mathbf{M}+\mathbf{H}]^{+} \text {ion }} \\
m / z\end{array}$ & $\begin{array}{l}\text { MSMS } \\
\text { Collision } \\
\text { Energy }\end{array}$ & Fragment ions $-m / z$ \\
\hline$\Delta^{7}$ mesembrenone & 287 & $\begin{array}{l}288.23 \\
\text { (1a) }\end{array}$ & $38 \%$ & $\begin{array}{l}288.05,273.05,232.75 \text { and } 149.47(\mathbf{1 b}) \\
\text { Proposed fragmentation pathway (1c) }\end{array}$ \\
\hline Mesembrenol & 289 & $\begin{array}{l}290.74 \\
\text { (1d) }\end{array}$ & $44 \%$ & $\begin{array}{l}290.70,272.71,241.76,229.65 \text { and } \\
210.60 \text { (1e) } \\
\text { Proposed fragmentation pathway (1f) }\end{array}$ \\
\hline Mesembranel & 291 & $\begin{array}{l}292.17 \\
(\mathbf{1 g})\end{array}$ & $31 \%$ & $\begin{array}{l}292.33,274.13 \text { and } 243.13 \text { (1h) } \\
\text { Proposed fragmentation pathway (1i) }\end{array}$ \\
\hline Mesembrenone & 287 & $\begin{array}{l}288.08 \\
(1 \mathbf{j})\end{array}$ & $28 \%$ & $\begin{array}{l}288.20,257.07 \text { and } 230.27(\mathbf{1 k}) \\
\text { Proposed fragmentation pathway ( 11) }\end{array}$ \\
\hline Mesembrine & 289 & $\begin{array}{l}290.17 \\
(1 \mathrm{~m})\end{array}$ & $37 \%$ & $\begin{array}{l}290.28,275.19,259.06,232.23 \text { and } \\
219.25 \text { ( 1n) } \\
\text { Proposed fragmentation pathway (10) }\end{array}$ \\
\hline Sceletium $\mathbf{A}_{4}$ & 324 & $\begin{array}{l}325.94 \\
\text { ( 2a) }\end{array}$ & $48 \%$ & $\begin{array}{l}325.89,294.84,282.86,268.84 \text { and } \\
187.69 \text { (2b) } \\
\text { Proposed fragmentation pathway ( } \mathbf{2 c})\end{array}$ \\
\hline Epimesembranol & 291 & $\begin{array}{l}292.23 \\
(\mathbf{1 p})\end{array}$ & $28 \%$ & $\begin{array}{l}291.70,273.74 \text { and } 242.75 \text { ( 1q) } \\
\text { Proposed fragmentation pathway (1r) }\end{array}$ \\
\hline
\end{tabular}

*All figure numbers appear in parenthesis in the above table.

Application of tandem mass spectroscopy provided similar data on the various chemical structures of each relevant alkaloid based on their characteristic ionic fragments and the proposed fragmentation patterns using EI as previously reported by Martin et al (10).

As an example, using Sceletium plant material, collision induced dissociation (CID) was successfully performed by tandem MS and the resulting product ions monitored as fingerprints of each of the six respective mesembrine-type alkaloids (Figure 3a-f).
The method was also successfully applied to various Sceletium species, processed plant material and its dosage forms and showed satisfactory ionization for identification of the relevant alkaloids (16). In addition to the previously mentioned six mesembrine-type alkaloids generally found in Sceletium plant species $(8,10)$, a non-mesembrine type alkaloid Sceletium $A_{4}$, which is a close analogue of tortuosamine previously described by Jeffs et al (17) as molecule found in Sceletium namaquense, in this instance Sceletium $\mathrm{A}_{4}$ has been found in Sceletium crassicaule (13) has been included. A specimen (SP 09 GRA) of Sceletium 
crassicaule species has been submitted to Selmar Schonland Herbarium, Rhodes University, Grahamstown, South Africa. This described method indeed provides the versatility of including other related compounds that occur in various Sceletium species (18).

A further important example of the application of this method involved the analysis of Sceletium plant material contained in tablet dosage forms (Sceletium tortuosum tablets - Big Tree Health Products, Cape Town, South Africa, Batch number
9332) and analyzed by HPLC-UV-MS which displayed the characteristic peaks for Sceletium alkaloids and were well resolved. However, the peak appearing at 5.50 minutes by UV detection (Figure $4 \mathrm{~d}$ ) was not $\Delta^{7}$ mesembrenone but one of the related alkaloids with an $\mathrm{m} / \mathrm{z}$ value of 273.96 (Figure 4a) as shown in the TIC chromatogram at RT 5.56 minutes (Figure 4b). However, the peak at RT of 5.72 by UV detection and 5.79 minutes by MS detection, displayed the $\mathrm{m} / \mathrm{z}$ 288.2, which corresponds to $\Delta^{7}$ mesembrenone (Figure $4 \mathrm{c}$ ).
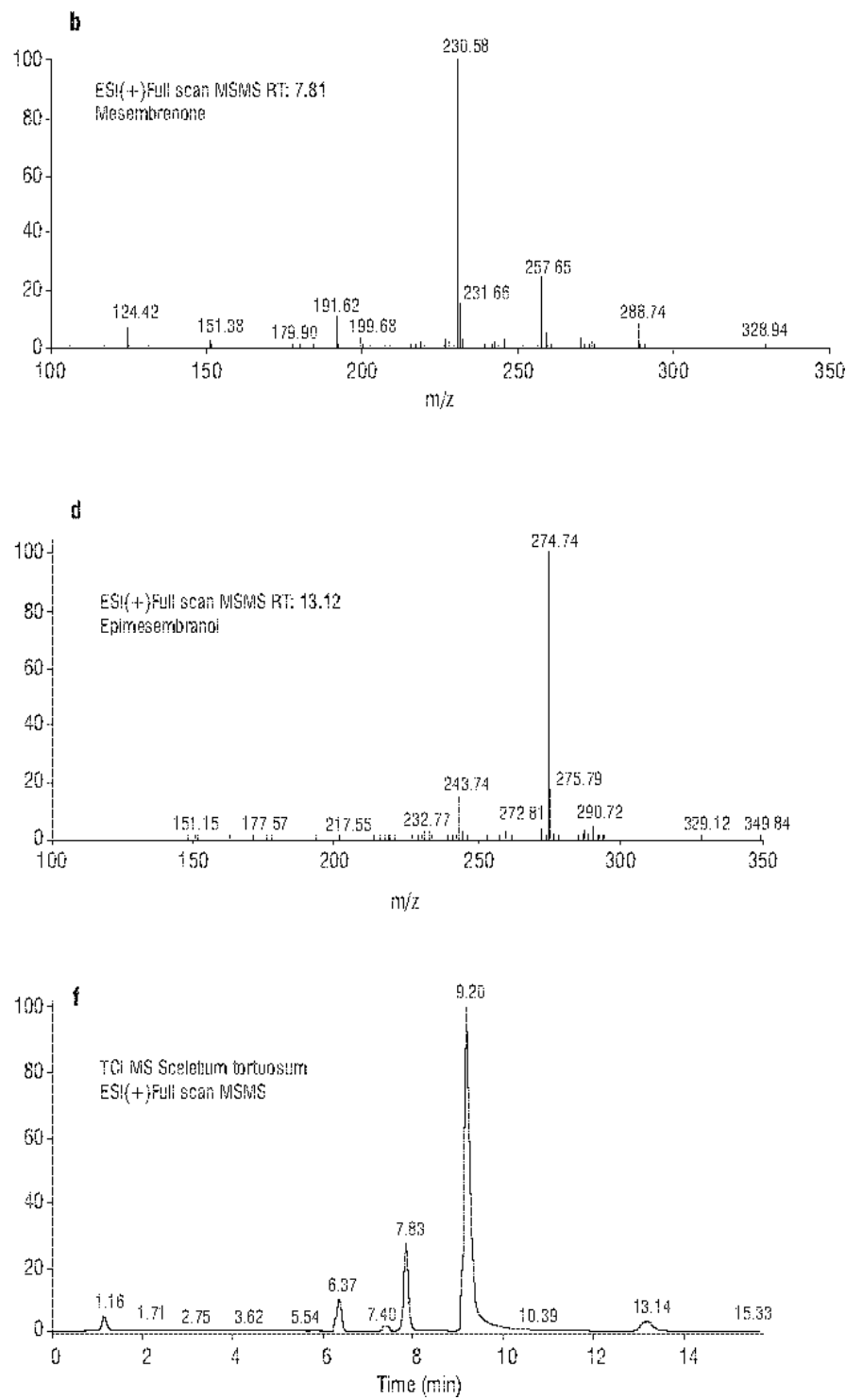

Figure 3a-f: Tandem MS profile Sceletium tortuosum plant material; $\Delta^{7}$ mesembrenone (3a); mesembrenone (3b); mesembranol (3c); epimesembranol (3d); mesembrine (3e); TIC full scan MS/MS (3f). 

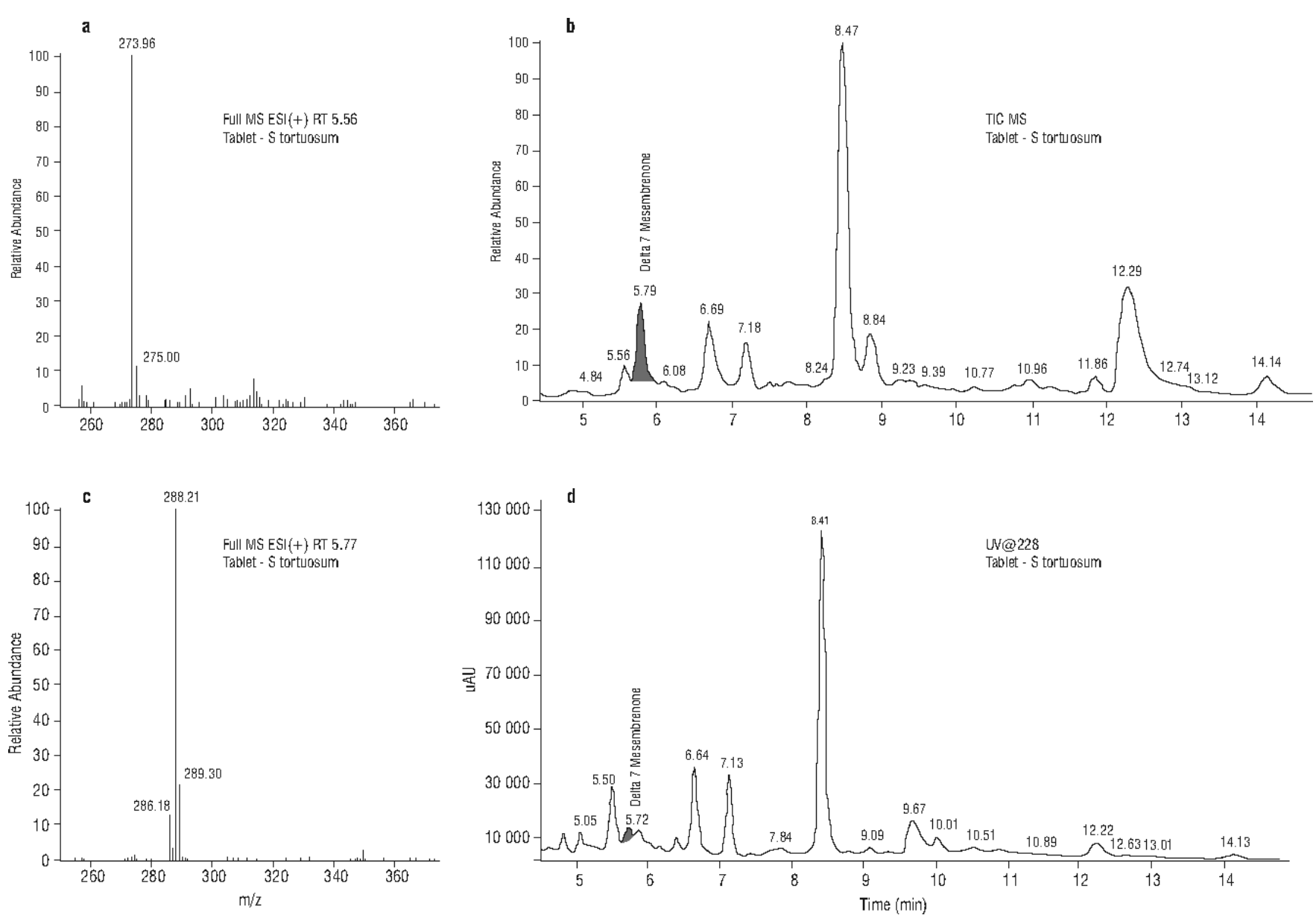

Figure 4a-c: Sceletium tortuosum tablets; Ion chromatogram for $m / z 273.96$ (4a); TIC MS chromatogram (4b); Ion chromatogram for $m / z 288.21$ (4c); UV detection (4d).

In another instance, a sample of Sceletium emarcidum (Figure 5d) when analyzed by HPLCUV-MS showed several peaks where some nonalkaloidal components eluted close to the RTs of the mesembrine reference alkaloid standards (Figure 5e). It is important to note that two of the resolved peaks in the TIC chromatogram at RT 4.01 and 13.53 minutes (Figure 5b) with $\mathrm{m} / \mathrm{z}$ ratios of 258.17 (Figure 5a) and 362.04 (Figure 5c), respectively, although ionized under the chromatographic conditions do not correspond to any of the reference standards, hence confirming the absence of any of the alkaloids, generally considered to be present in most of the other Sceletium species, in the sample.

Importantly, the described LC-MS method has shown high sensitivity for identification of mesembrine-type alkaloids with an LoQ for mesembrine of $50 \mathrm{ng} / \mathrm{ml}$ (16) compared to the reported GC-MS (11) methodology where the LoQ was approximately 1000 times less sensitive $(68.3$ $\left.\mu \mathrm{g} \mathrm{ml}^{-1}\right)$. In addition, unlike the previously reported HPLC-UV method (8), the RP-UHPLC method of Shikanga et al (11) does not include the identification and quantification of $\Delta^{7}$ mesembrenone, $\quad$ Sceletium $\quad \mathrm{A}_{4}$ or epimesembranol.

\section{DISCUSSION}

Use of herbal remedies is on the rise and especially so in the treatment of psychiatric disorders and related mental health conditions (19). Hence, these remedies have to be studied in depth to understand the implications of the risks, side effects and contraindications that may be associated with such preparations. 

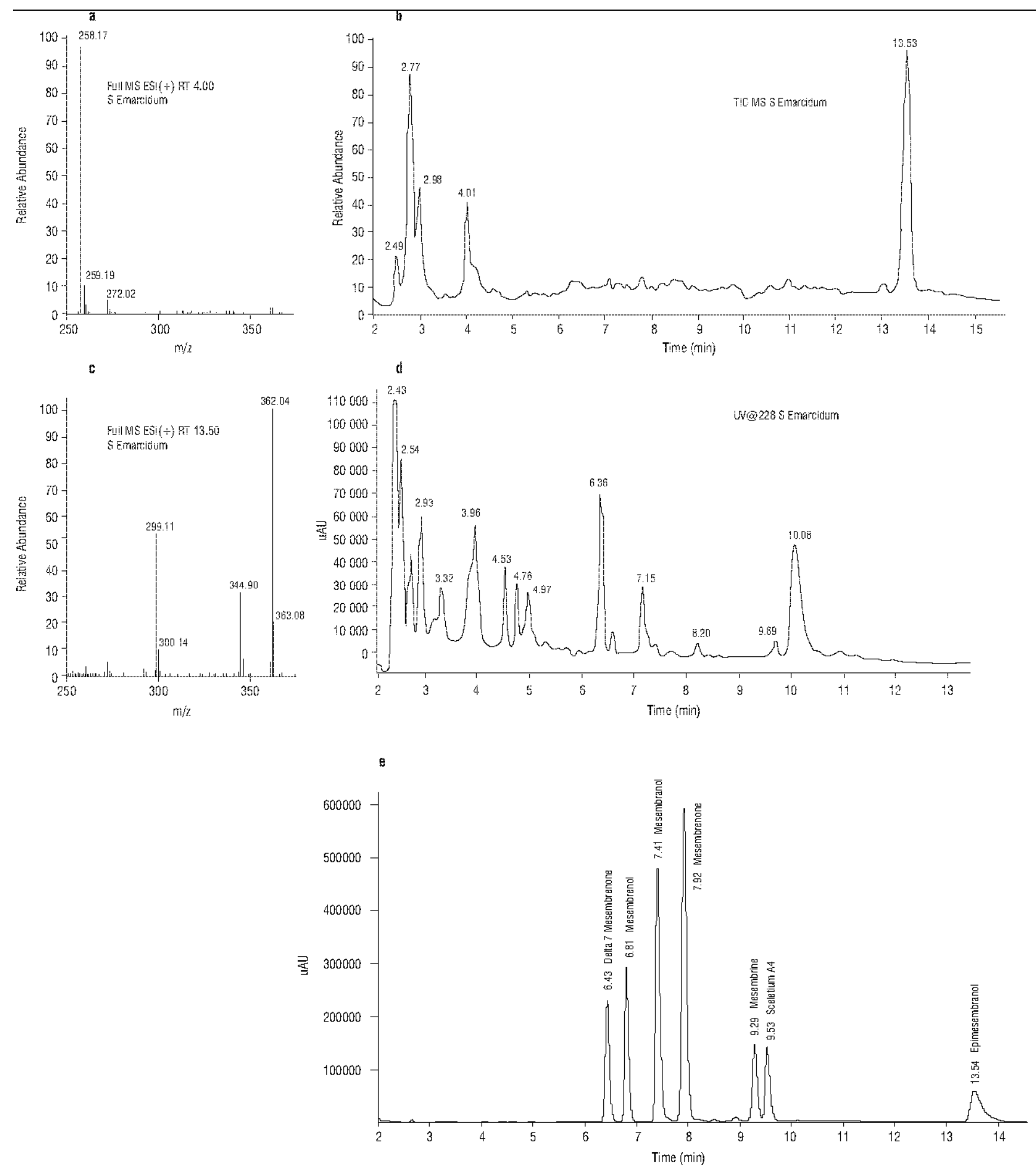

Figure 5a-c: Sceletium emarcidum plant sample; Ion chromatogram for $m / z 258.17$ (5a); TIC chromatogram (5b); UV detection (5d); Ion chromatogram for $m / z 362.04$ (5c); Sceletium reference alkaloid standards (5e).

Sceletium species have been claimed to be effective in cases of depression which have been linked to inhibitory effects on 5-HT reuptake (19). However, since several of these mesembrine-type alkaloids may or may not be present in different Sceletium plant species (18) and given the closeness of their chemical structures, it is indeed a challenge to identify and confirm the specific components 
existing not only in plant species but also in the associated products.

LCMS is fast becoming the method of choice for the analysis of natural compounds due to its unique ability to identify the compounds by their molecular masses and characteristic fragmentation pattern. The efficiency of the LCMS method was enhanced by development of a satisfactory HPLCUV method for the chromatographic separation and quantitative analysis (8) of the relevant alkaloids. The ionization method used also enhanced the sensitivity, which was found to be selective for Sceletium alkaloids. Since the identified markers have closely related structures, the chromatographic separation of these related alkaloids provided specificity for the unequivocal identification of the alkaloids based on their $m / z$ ratios.

The current procedures facilitated the fingerprinting and qualitative assessment and identity of the various Sceletium alkaloids. Furthermore, this LCMS method was found to efficiently ionize the alkaloidal markers amongst the complex components present in plant material and in tablet dosage forms without apparent interference from excipients present in those formulations.

Various species of Sceletium plants were subsequently provided by Sceletium plant cultivators and this LCMS method was successfully used for chemotaxonomy of some Sceletium species (18) and commercially available Sceletium dosage forms. More importantly this method proved valuable to monitor the fermentation process of Sceletium plant material (9). Hence the current qualitative LCMS method, and concurrent application of the previously reported quantitative assay method (8), provides valuable analytical procedures for the identification and QC of Sceletium plant material and its dosage forms. An additional advantage of the LC- application of ESIMS tandem mass spectroscopy provided unique fragmentation patterns to specifically identify each alkaloid in complex matrices, thereby providing a valuable confirmatory method for detection and quantification in such diverse matrices.

Finally, as previously indicated, use of conventional HPLC-UV, although providing useful information with respect to retention times, can result in erroneous identification of isobaric and isomeric compounds and also epimers where such chemical structures are either closely related, or similar as in the case of epimers, and structural differences may involve only differences in conformation or configuration. Generally, HPLC using UV detection cannot discriminate between such compounds and hence detection by MS enhances the accuracy and specificity of the analytical method thereby reducing the risk of using an inappropriate Sceletium species for the indications on the product label.

\section{ACKNOWLEDGEMENTS}

Funding from the Biopharmaceutics Research Institute, Rhodes University, Grahamstown South Africa, is gratefully acknowledged.

\section{CONFLICT OF INTEREST}

The authors declare that they have no conflict of interest.

\section{REFERENCES}

1. Smith MT, Crouch NR, Gericke N, Hirst M. Phychoactive constituents of the genus Sceletium N.E.Br. and other Mesembryanthemaceae: a review. J Ethanopharmacol, 1996; 50:119-130.

2. Smith MT, Field CR, Crouch NR, Hirst M. The distribution of Mesembrine alkaloids in selected Taxa of the Mesembryanthemaceae and their modification in the Sceletium derived 'Kougoed'. Pharm Biol, 1998; 36(3):173-179.

3. Murbach TS, Hirka G, Szakonyiné P I, Gericke N, Endres J R. A toxicological safety assessment of a standardized extract of Sceletium tortuosum $\left(\right.$ Zembrin $\left.^{\circledR}\right)$ in rats. Food Chem Toxicol, 2014; 74:190-199.

4. Smith C, The effects of Sceletium tortuosum in an in vivo model of psychological stress. J Ethnopharmacol, 2011; 133: 31-36

5. Mauri P, Pietta P. Electrospray characterization of selected medicinal plant extracts. J Pharm Biomed Anal, 2000; 23(1): 61-68.

6. Nicolas EC, Scholz TH. Active drug substance impurity profiling part II LC/MS/MS fingerprinting. J Pharm Biomed Anal, 1998; 16: 825-836.

7. Roscher J, Posch NT, Putz M, Huhn C. Forensic analysis of mesembrine alkaloids in Sceletium tortuosum by nonaqueous capillary electrophoresis mass spectrometry. Electrophoresis, 2012, 33, 15671570.

8. Patnala S, Kanfer I. HPLC analysis of Mesembrinetype alkaloids in Sceletium plant material used as an African traditional medicine. J. Pharm. Pharm. Sci, 2010; 13(4): 558-570. 
9. Patnala S, Kanfer I. Investigation of phytochemical content of Sceletium tortuosum following the preparation of "Kougoed" by fermentation of plant material. J Ethnopharmacol, 2009; 121: 86-91.

10. Martin NH, Rosenthal D, Jeffs PW. Mass Spectra of Sceletium Alkaloids. Org Mass Spec, 1976;11:1-19.

11. Shikanga EA, Kamatou GPP, Chen W, Combrinck S, Viljoen AM. Validated RP-UHPLC PDA and GC-MS Methods for the analysis of psychoactive alkaloids in Sceletium. S Afr J Bot, 2012; 82: 99107

12. Gericke N, Viljoen AM. Sceletium - a review update. J Ethnopharmacol, 2008; 119: 653-663

13. Patnala S, Kanfer I. Unpublished data. 2015

14. Bodendorf K, Krieger W. Über die alkaloide von Mesembryanthemum tortuosum. L Arch Pharm, 1957; 290/62(10): 441-448.

15. Jeffs PW, Hawks RL, Farrier DS. Structure of mesembranols and the absolute configuration of mesembrine and related alkaloids. J Am Chem Soc, 1969; 91(14): 3831.

16. Patnala S. Pharmaceutical analysis and quality of complementary medicines: Sceletium and associated products (Thesis). Grahamstown: Rhodes University, 2007

17. Jeffs PW, Luhan PA, McPhail AT, Martin NH. The structure of Sceletium alkaloid A4, a pyridine alkaloid from Sceletium namaquense: Direct method X-ray determination. Chem Com, 1971; 1343: 14661467.

18. Patnala S, Kanfer I. Chemotaxonomic studies of mesembrine-type alkaloids in Sceletium plant species. S Afr J Sci, 2013; 109(3/4): 882-886.

19. Gericke NP, Van Wyk B-E. Pharmaceutical compositions containing mesembrine and related compounds. 2001; US Patent 6,288,104 B1. 